\title{
DNA Genetic Optimization Bat Algorithm Based Fractionally Spaced Multi-modulus Blind Equalization Algorithm
}

\author{
Huapeng $W^{a}{ }^{a}$, Yecai Guo \\ College of Electronic \& Information Engineering, Nanjing University of Information Science \& \\ Technology, Nanjing, 210044, China \\ aemail: guo-yecai@163.com, bemail: 61892133@qq.com
}

Keywords: Fractionally spaced equalizer(FSE); Multi-modulus blind equalization algorithm(MMA); DNA genetic optimization bat algorithm(DNA-GBA); Global optimal position

\begin{abstract}
A DNA genetic optimization bat algorithm based fractionally spaced multi-modulus blind equalization algorithm (DNA-GBA-FS-MMA) is proposed. This proposed algorithm uses fractionally spaced equalizer(FSE) with ability to oversample to input signals to get more channel information and compensate for channel with the depth spectrum zero, employs DNA genetic optimization bat algorithm(DNA-GBA) for searching the global optimal position vector, which are simultaneously regarded as the real and imaginary parts of the initial weight vector of multi-modulus blind equalization algorithm(MMA) in order to improve convergence speed and reduce mean square error(MSE). Simulation results show that DNA-GBA-FS-MMA has the best equalization effect.
\end{abstract}

\section{Introduction}

Multi-modulus blind equalization algorithm(MMA) can simultaneously carry out the function of blind equalization and carrier recovery in the absence of the carrier-recovery system[1]. Fractionally spaced equalizer(FS) combined with the MMA can reduce the mean square error (MSE) and the calculation load via oversampling to the input signals[2], but its convergence speed is still slow. DNA genetic algorithm(DNA-GA) uses DNA molecular operation to improve crossover, mutation, and selection operations of the genetic algorithm(GA), as well as strong global search ability, whereas the echo location characteristics of bat algorithm (BA) can avoid falling into local searching of the searching process and improve the success rate of searching global optimal position vector.

In this paper, after we introduce DNA-GA into BA, a DNA genetic optimization bat algorithm based fractionally spaced multi-modulus blind equalization algorithm(DNA-GBA-FS-MMA) is proposed to search the global optimal position vector, which is used to optimize the weight vector of the MMA. Simulation results verify the effectiveness of DNA-GBA-FS-MMA algorithm.

\section{DNA Genetic Optimization Bat Algorithm Based Fractionally Spaced MMA}

When we introduce the DNA-GA into the BA, the DNA-GA based BA is called as DNA genetic optimization bat algorithm(DNA-GBA). When we introduce the DNA-GBA and the MMA into fractionally spaced equalizer, DNA genetic optimization bat algorithm based fractionally spaced multi-modulus blind equalization algorithm(DNA-GBA-FS-MMA) is obtained and shown in Figure 1. Fig.1(a) is a principle of the fractionally spaced MMA(FS-MMA), Fig.1(b) corresponds to the MMA module in Fig.1(a).In the MMA module, the $\boldsymbol{w}_{R}(k)$ and $\boldsymbol{w}_{I}(k)$ are updated as follows:

$$
\left\{\begin{array}{l}
\boldsymbol{w}_{R}(k+1)=\boldsymbol{w}_{R}(k)-4 \mu e_{R}(k) \boldsymbol{y}_{R}(k) \\
\boldsymbol{w}_{I}(k+1)=\boldsymbol{w}_{I}(k)-4 \mu e_{I}(k) \boldsymbol{y}_{I}(k)
\end{array}\right.
$$

where $\mu$ is the step-size, $0 \leq \mu \leq 1$.

To simplify calculation and reduce the MSE, we introduce T/2 FSE into MMA to obtain more detailed channel information and compensate the fuzzy channels better. FSE based multi-modulus algorithm(FS-MMA) can decrease the MSE, but its convergence speed is still slow. 
For the purpose of accelerating convergence speed and reducing the MSE, we use the BA to

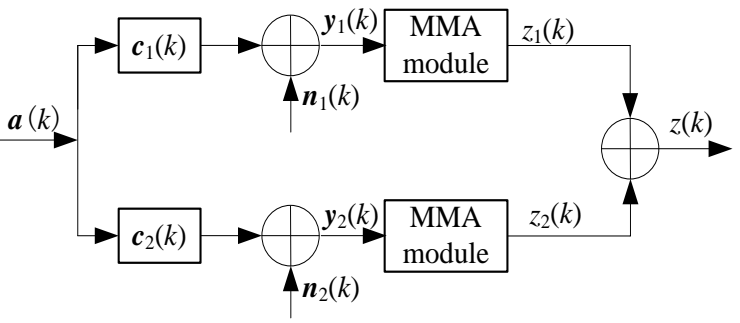

(a)

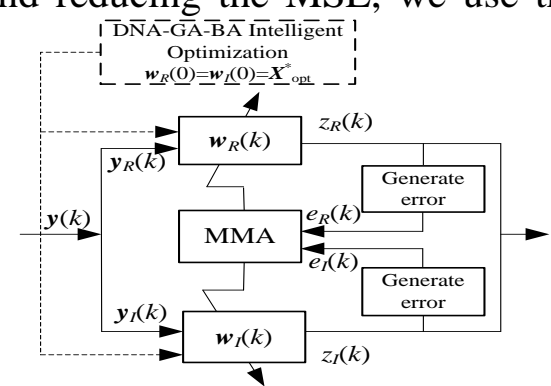

(b)

Fig.1 DNA Genetic Optimization Bat Algorithm Based Fractionally Spaced Equalizer Multi-modulus Blind Equalization Algorithm

search the global optimal position vector, which is served as the real and imaginary parts of the initial weight vector simultaneously. The velocity $\boldsymbol{V}_{i}(k)$ and position $\boldsymbol{X}_{i}(k)$ of the bat are updated as

$$
\begin{aligned}
& f_{i}=f_{\min }+\left(f_{\max }-f_{\min }\right) \beta \\
& \boldsymbol{V}_{i}(k)=\boldsymbol{V}_{i}(k-1)+\left[\boldsymbol{X}_{i}(k)-\boldsymbol{X}^{*}\right] f_{i} \\
& \boldsymbol{X}_{i}(k)=\boldsymbol{X}_{i}(k-1)+\boldsymbol{V}_{i}(k)
\end{aligned}
$$

where $\beta \quad \notin 0,1]$ and obeys uniform distribution. $\boldsymbol{X}^{*}$ is the current global best position of the whole bat swarm. $f_{\mathrm{i}}$ is frequency and is used to control the update of $\boldsymbol{V}_{i}(k)$.

In local searching, once a position $\boldsymbol{X}_{\text {old }}$ from the current best positions of all bats is selected, then the new position $\boldsymbol{X}_{\text {new }}$ of the bat swarm is generated by a random walk from $\boldsymbol{X}_{\text {old }}$ [4].

$$
\boldsymbol{X}_{\text {new }}=\boldsymbol{X}_{\text {old }}+\varepsilon A(k)
$$

where $\varepsilon \quad[\in, 1]$ is a random number, $A(k)$ is average loudness of all bats. $\boldsymbol{X}_{\text {new }}$ is used to replace $\boldsymbol{X}_{i}(k)$ to make the searching process go back to the global searching and avoid running into local optimal.

During the searching process, bats emit ultrasonic with large loudness $A$ and low frequentness $r$ to find prey in broader area at beginning. Once they find their target, the loudness $A$ will decrease and frequentness $r$ will raise to search more precise. So we have

$$
\begin{aligned}
& \boldsymbol{A}_{i}(k+1)=\alpha \boldsymbol{A}_{i}(k) \\
& r_{i}(k+1)=r(0)[1-\exp (-\gamma k)]
\end{aligned}
$$

where $\alpha$ and $\gamma$ are loudness and frequentness attenuation coefficient, respectively. Frequentness and loudness change when best position changes. That means all bats move toward new best position.

For improving efficiency of searching global optimal position, crossover and mutation operations of DNA-GA are used to optimize the searching process of the BA. The combination of the DNA-GA and the BA-FS-MMA can further improve the convergence speed, reduce the MSE, and make up for defects of the low searching efficiency and falling into local convergence of GA. DNA-GA has not only strong global searching ability, self-organizing, self-adaptive, and self-learning ability of the GA, but also its own unique advantages. It has 4 kinds of bases $\mathrm{A}, \mathrm{G}, \mathrm{C}$, and $\mathrm{T}$, which can be denoted by the four hexadecimal code such as $0,1,2$, and 3 . There are two complementary pairs such as A-T and C-G, which corresponds to 0-3 and 1-2 after encoding. This encoding increases the diversity of the population, solves the Hamming cliffs, and improves genetic operations. The object function of the DNA-GBA-FS-MMA is defined as

$$
J\left(\boldsymbol{X}_{i}(k)\right)=E\left\{\left[z_{R}^{2}(k)-R_{R}^{2}\right]^{2}+\left[z_{I}^{2}(k)-R_{I}^{2}\right]^{2}\right\}
$$

where $\boldsymbol{X}_{i}(k)$ corresponds to $\boldsymbol{w}_{R}(k)$ and $\boldsymbol{w}_{I}(k)$ simultaneously in the MMA module, $z_{R}(k)=\boldsymbol{X}_{i}(k) \cdot \boldsymbol{y}_{R}(k)$ and $z_{I}(k)=\boldsymbol{X}_{i}(k) \cdot \boldsymbol{y}_{I}(k)$. Implementation steps of the DNA-GBA-FS-MMA are as follows:

Step 1: Initial the parameters. Generate a random bat swarm, bat number $n$, frequency $f_{\mathrm{i}} \in\left[f_{\min }\right.$, $\left.f_{\max }\right]$, permutation crossover probability $p_{z}$, translocation crossover probability $p_{y}$, maximum frequentness $r(0)$, loudness attenuation coefficient $\alpha$, frequentness attenuation coefficient $\gamma$, 
maximum loudness $A(0)$, and the position of each bat $\boldsymbol{X}_{i}$.

Step 2: Calculate the object function values according to Eq.8 and find their minimum, the position corresponding to minimum is the current global optimal position $\boldsymbol{X}^{*}$.

Step 3: Generate new positions via adjusting frequency, updating velocities and positions[4] with Eq.4, then we obtain the updated positions $\boldsymbol{X}_{i}(k)$.

Step 4: Compare the current frequentness $r_{i}$ with a random rate rand1. If $r_{i}>$ rand1, a new position besides current optimal position is generated, local searching process returns to global searching.

Step 5: Compare the current loudness $A_{i}$ with a random loudness rand2. If $A_{i}<$ rand 2 and $J\left(\boldsymbol{X}_{i}(k)\right)<J\left(\boldsymbol{X}^{*}\right), \boldsymbol{X}^{*}$ is replaced with $\boldsymbol{X}_{i}(k)$ and $A_{i}$ and $r_{i}$ are updated by Eq.6 and Eq.7, respectively.

Step 6: DNA base encoding. Each position of the bat swarm is encoded with the special DNA base encoding, then a group of position DNA sequences are obtained.

Step 7: Permutation crossover. Two position DNA sequences which selected randomly are regarded as parent bodies. permutation crossover probability $p_{z}$ is compared with a random number rand3, if rand $3 p_{z}$, two equal number bases sequences will be selected from each parent bodies to exchange, then two new position DNA sequences are generated to replace the parent bodies.

Step 8: Transposition crossover. One position DNA sequence which selected randomly is regarded as parent body. Comparing translocation crossover probability $p_{y}$ with a random number rand4, if rand $4<p_{y}$, a DNA sequence is cut down from the parent body and inserted in optional position of the rest parent body sequence. Then a new position DNA sequence is generated to replace the parent body.

Step 9: Mutation operator. One position DNA sequence which selected randomly is regarded as parent body, a new position sequence is generate by replacing the base appearing most frequent with the base in lowest frequency to take the place of the parent body.

Step 10: Decoding operator. To calculate the object function values and find the minimum, all position DNA sequences are decoded to a new group of position vectors.

Step 11: Choose the current global optimal position $\boldsymbol{X}^{*}$.

Step 12: Achieve the global optimal position. When reaching the the searching accuracy or maximum iteration number, the global optimal position $\boldsymbol{X}^{*}$ is regarded as $\boldsymbol{X}_{\text {opt }}{ }^{*}$. Otherwise, the searching process turns to step 3.

Step 13: Achieve the original weight vector. $\boldsymbol{X}_{\text {opt }}{ }^{*}$ is used as the real and imaginary parts of the weight vector $\boldsymbol{w}(k)$, meanwhile. That is, $\boldsymbol{w}_{R}(0)=\boldsymbol{w}_{I}(0)=\boldsymbol{X}_{\text {opt }}{ }^{*}$. After updating $\boldsymbol{w}_{R}(k)$ and $\boldsymbol{w}_{I}(k)$ with Eq.1, then input signals of the two branch can be equalized more effectively.

\section{Test results}

To verify the performance of DNA-GBA-FS-MMA, the simulation tests were compared with MMA, FS-MMA, BA-MMA, DNA-GBA-MMA, and BA-FS-MMA. In simulations, 16QAM signals were transmitted, the step-size $\mu_{\mathrm{MMA}}=\mu_{\mathrm{FSE}-\mathrm{MMA}}=0.018, \mu_{\mathrm{BA}-\mathrm{MMA}}=\mu_{D N A-G A-B A-\mathrm{MMA}}=0.0017$, $\mu_{\text {BA-FSE-MMA }}=\mu_{D N A-G A-B A-F S E-M M A}=0.003$. The other parameters were given as foloows: $n=20, A(0)=1.5$, $r(0)=0.25, f_{\mathrm{i}} \in[0,100], \alpha=\gamma=0.9, p_{z}=0.8, p_{y}=0.3$, and channel $\boldsymbol{c}=\left[\begin{array}{ll}0.9556 & -0.09060 .05780 .2368\end{array}\right]$. Simulation results are shown in Fig.2. Fig.2(a) is the curves of the MSE, Fig.2(b) (g) are the output constellations of the MMA, the BA-MMA, the FS-MMA, the DNA-GBA-MMA, the BA-FS-MMA, and the DNA-GBA-FS-MMA.

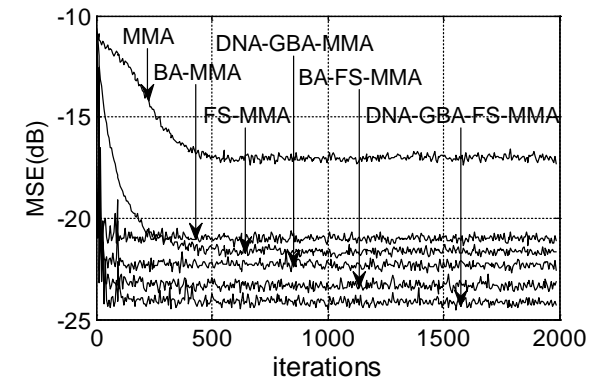

(a)

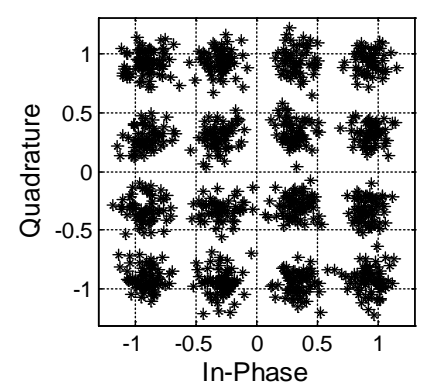

(b)

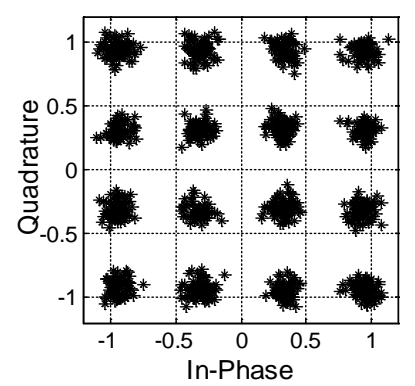

(c) 


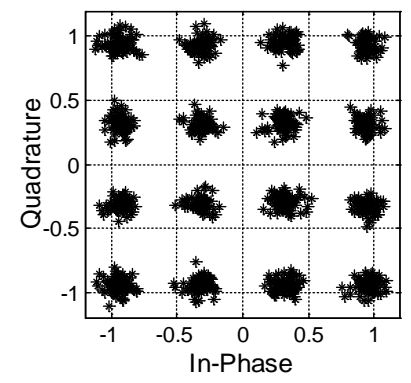

(d)

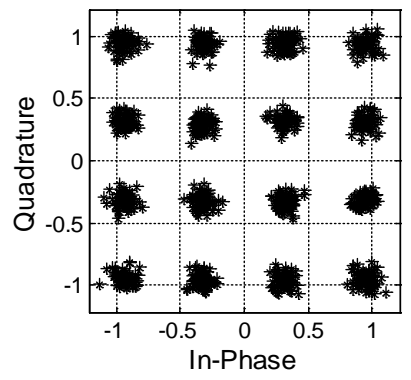

(e)

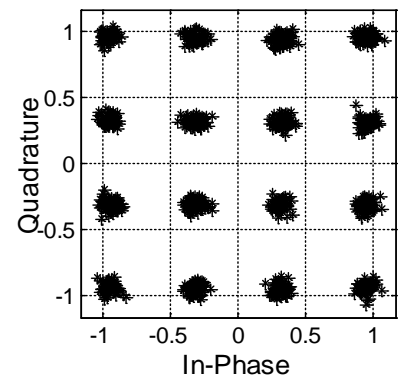

(f)

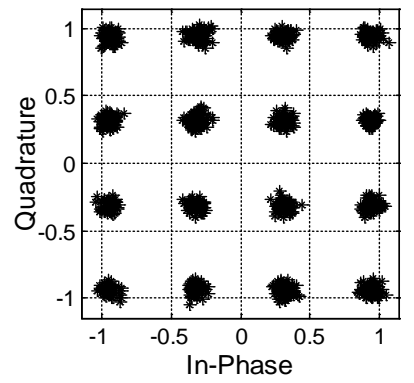

(g)

Fig.2 Mean Square Error Curve and constellations

From Fig.2, we can know that DNA-GBA-FS-MMA has the best equalization effect, the MSE reaches $-24 \mathrm{~dB}$, and its constellations are the clearest and the most compact comparison with the MMA, the FS-MMA, the BA-MMA, the DNA-GBA-MMA, and the BA-FS-MMA.

\section{Conclusions}

A DNA genetic optimization bat algorithm based fractionally spaced equalizer multi- modulus blind equalization algorithm(DNA-GBA-FS-MMA) is proposed in this paper. This proposed algorithm can accelerate the convergence speed, reduce the MSE, and correct phase rotation. Therefore, the proposed DNA-GBA-FSE-MMA is feasible.

\section{Acknowledgment}

This work is supported by the major project of University Natural Science of Jiangsu Province (No.13KJA510001), Jiangsu Scientific Research Achievements in Industrialization Project (JHB 2012-9), and A Project Funded by the Priority Academic Program Development of Jiangsu Higher Education Institutions(PAPD II), as well as Innovation Project of Postgraduate Training in Jiangsu Province(No. SJZZ_0110, SJZZ_0111, and KYLX_0853).

\section{References}

[1] Y. Jenqtay, T. Kunda: Analysis of the Multimodulus Blind Equalization Algorithm in QAM Communication Systems. IEEE. Vol.53(2005), p.1427.

[2] G. Yeicai, D. Xuejie and F. Kang:Fractionally Spaced Combining with Spatial Diversity Blind Equalization Algorithm Based on Orthogonal Wavelet Transformation. Intelligent Computing and Cognitive Informatics (2010).

[3] Y.Jian, J.J.Werner and G.A.Dumont: The Multimodulus Blind Equalization and Its Generalized Algorithms. IEEE . Vol. 20(2002), p. 997.

[4] Y.Xinshe, in: Nature Inspired Cooperative Strategies for Optimization (NISCO), edited by J.R.Gonzalez, Springer, Berlin(2010).

[5] W. Mingchun, T. Wansheng and L.Xin: The DNA Genetic Algorithm Applied for Solving Stochastic Integer Programming Expected Value Models. Machine Learning and Cybernetics(2008). 